\title{
Assessment of Osteoporosis in Post Menopausal Women: A Clinical Study
}

\author{
Najam R', Huda $N^{2}$ \\ 'Department of Obs/Gyn, Teerthankar Mahaveer Medical College, ${ }^{2}$ Department of Orthopedics, Teerthankar \\ Mahaveer Medical College, Moradabad, U.P.
}

\begin{abstract}
Aims: Osteoporosis is a metabolic disorder of the bones that is characterized by decreased bone density which predisposes to increased fracture risk. The disease can exist undiagnosed for a long time as it produces no distinct symptoms and is detected when a fracture has already occurred. There is sufficient evidence to state that the risk for osteoporosis and fracture increases with age and after menopause, that bone density measurements accurately predict the risk for fractures in the short-term, and that treating asymptomatic women with osteoporosis reduces their risk for fracture. The present cross sectional study was undertaken to assess osteoporosis in postmenopausal women by measuring bone mineral density (BMD).

Methods: This is a prospective study, conducted at the Department of Obstetrics and Gynecology, Teerthanker Mahaveer Medical College, Moradabad. India. Post menopausal women were included in the study. Women who had undergone hysterectomy or who were on hormone replacement therapy or calcium or vitamin D preparations were excluded from the study. The participants underwent BMD measurement by Achilles Ultrasound bone densitometer. The results of BMD were analysed on the basis of T-Scores and were represented in the form of a graph.

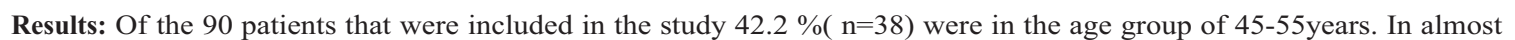
$50 \%$ of patients the duration of menopause was $6-10$ years. Of the 90 patients $10 \%(n=9)$ were normal, $44.4 \%(n=40)$ were osteopenic, $33.3 \%(\mathrm{n}=30)$ were osteoporotic and $12.2 \%(\mathrm{n}=11)$ were severely osteoporotic. Backache was the commonest presenting symptom in the study population.

Conclusions: Quantitative ultrasound (QUS) is a rapid and inexpensive method of measuring bone density. In our study $90 \%$ of the postmenopausal women had subnormal T scores. We recommend regular BMD measurements in postmenopausal subjects for early diagnosis and treatment of osteoporosis in this vulnerable group.
\end{abstract}

Keywords: Bone mineral density, osteoporosis, postmenopausal, quantitative ultrasound

\section{INTRODUCTION}

Osteoporosis is a metabolic disorder of the bones that is characterized by decreased bone density which predisposes to increased fracture risk. ${ }^{1,2}$ The disease can exist undiagnosed for a long time as it produces no distinct symptoms and is detected when a fracture has already occurred. Hence it is also known as a "silent epidemic". It is estimated that 28 million Americans suffer from bone loss and of these 10 million have established osteoporosis. ${ }^{3}$ A woman left untreated has a $50 \%$ chance of suffering from a osteoporotic fracture. ${ }^{4}$ Johnell in a study on the socio economic burden of fractures states that the cost of hip fractures imposes a significant economic burden on society. The projected cost of hip fractures in the year 2050 is $\$ 131.5$ billion. ${ }^{5}$ Thus it is of utmost importance to detect the disease early and treat it. Fracture risk increases significantly with age and the incidence rises after the menopause. The United States preventive services task force (USPSTF) found good evidence that the risk for osteoporosis and fracture increases with age and other factors, that bone density measurements accurately predict the risk for fractures in the short-term, and that treating asymptomatic women with osteoporosis reduces their risk for fracture. The USPSTF concludes that the benefits of screening and treatment are of at least moderate magnitude for women at increased risk by virtue of age or presence of other risk factors. ${ }^{6}$ Osteoporosis is most commonly diagnosed using bone densitometry. The T-score is the number of standard deviations the bone

\section{CORRESPONDENCE}

Dr. Rehana Najam, MS

Department of Obstetrics and Gynaecology

Teerthankar Mahaveer Medical College and University,

Near Pakbara town, Moradabad, U.P.

Mobile no: 09837291920

Email: najamnajam@rediffmail.com 
mineral density (BMD) is below or above the mean of a young control group. It is used to diagnose osteoporosis. The Z-scores compare the BMD with the age and sex matched controls. Osteoporosis is defined as a T-score equal to or less than -2.5 , any T-score above this but below -1.0 is labeled osteopenia. (Table 1 )

Various techniques are available to measure bone density, but to date dual energy X-ray absorptiometry (DXA) scan is the best predictor. Many studies have evaluated the role of quantitative ultrasonographic (QUS) measurement of BMD at the heel. ${ }^{7,8}$ Several other radiologic methods that measure BMD at peripheral sites are single photon absorptiometry, quantitative computed tomography, single energy X-ray absorptiometry, and peripheral quantitative computed tomography. ${ }^{9}$

The risk factors for osteoporosis are advancing age, menopause whether natural or surgical, white or Asian ethnicity, history of fracture, family history of osteoporotic fracture, history of falls, low levels of physical activity, smoking, excessive use of caffeine or alcohol, low calcium or vitamin D intake and various medications.

The present study was undertaken for assessment of osteoosis in a cross section of postmenopausal women.

\section{METHODS}

This was a prospective study that was conducted in the Department of Obstetrics and Gynecology of our institute. Women who had attained menopause

Table 1. WHO criteria for assessing disease severity

\begin{tabular}{ll}
\hline Diagnostic classification & T-score \\
\hline Normal & $>-1.0$ \\
Osteopenia & -1.0 to-2.5 \\
Osteoporosis & $<=-2.5$ \\
Severe osteoporosis & $<-2.5$ with fracture \\
\hline
\end{tabular}

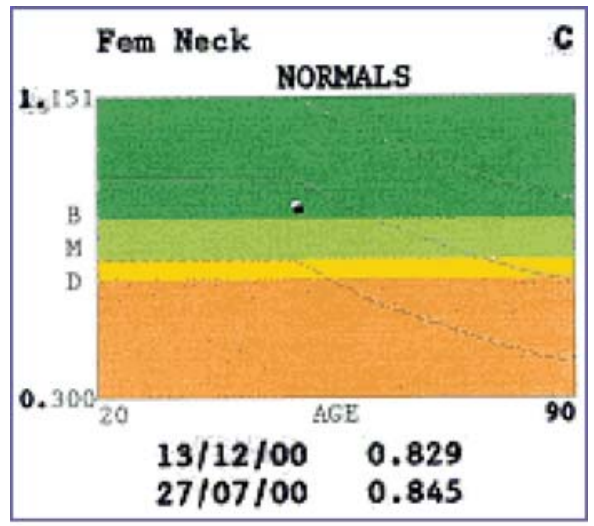

Bone Density Chart scale

Figure 1. BMD result graph were included in the study. Women who had undergone hysterectomy or who were on hormone replacement therapy or were taking calcium or vitamin D preparations were excluded from the study. All the participants were subjected to BMD measurements by the Achilles Ultrasound bone densitometer. Quantitative ultrasound (QUS) is an easy and inexpensive modality to measure BMD. The parameters measured are speed of sound (SOS) and broadband ultrasound attenuation (BUA) at the os calcis. BMD was assessed as stiffness index. The results of BMD were analysed on the basis of T-Scores and were represented in the form of a graph. T-score is the the number of standard deviations above or below the mean for a healthy 20 year old adult of the same sex and ethnicity as the patient.

The area of the graph labeled green shows the stiffness value of young adults and is considered normal (Figure 1). The area shaded light green shows $T$ score 1 standard deviation (SD) below the normal. The yellow region of the graph depicts T-score is 1-3 SD below the young healthy adult. The red portion represents values $>3$ SD lesser than the normal.

Data was collected regarding the age, duration of menopause, socio economic status, symptoms suggestive of low BMD.

\section{RESULTS}

Of the 90 patients that were included in the study $42.2 \%($ $n=38$ ) were in the age group of $45-55$ years (Table 2 ).

Table 2. Age distribution of study group

\begin{tabular}{lll}
\hline $\begin{array}{l}\text { Age-group } \\
\text { (years) }\end{array}$ & Number & Percentage \\
\hline $45-55$ & 38 & 42.3 \\
$56-65$ & 25 & 27.7 \\
$66-75$ & 12 & 13.3 \\
$76-85$ & 11 & 12.2 \\
$>85$ & 4 & 4.5 \\
\hline Total & 90 & 100 \\
\hline
\end{tabular}

In almost $50 \%$ of patients the duration of menopause was 6-10 years (Table 3 ).

Table 3. Distribution of patients according to the duration of menopause

\begin{tabular}{lcc}
\hline Duration (years) & Number & Percentage \\
\hline $1-5$ & 17 & 18.8 \\
$6-10$ & 46 & 51.2 \\
$>10$ & 27 & 30.0 \\
\hline Total & 90 & $\mathbf{1 0 0}$ \\
\hline
\end{tabular}


Of the 90 patients $10 \%(n=9)$ were normal, $44.4 \%(n=40)$ were osteopenic, $33.3 \%(n=30)$ were osteoporotic and $12.2 \%(n=11)$ were severely osteoporotic. Backache was the commonest presenting symptom in the study population. It was largely seen in osteopenic and osteoporotic females. Polyarthralgia was seen in $26.6 \%(n=24)$ patients. Kyphosis was seen in $10 \%$ of cases (Table 4 )

\section{Table 4. Distribution of patients according to the presenting symptom}

\begin{tabular}{lll}
\hline Symptom & Number & Percentage \\
\hline Backache & 57 & 63.3 \\
Polyarthralgia & 24 & 26.6 \\
Kyphosis & 9 & 10.0 \\
\hline Total & 90 & 100 \\
\hline
\end{tabular}

\section{DISCUSSION}

Osteoporosis is a metabolic bone disorder characterized by decreased bone density and deterioration in micro architectural framework of bone leading to fragility fractures. ${ }^{10}$ With the increase in the geriatric population worldwide osteoporosis is the commonest bone problem of the elderly. Due to this demographic change it is estimated that the risk of hip fractures will increase approximately up to 6 folds till 2050. ${ }^{11}$ The magnitude of the problem is such that a women's lifetime risk of hip fracture is equal to the combined risk of breast, uterine and ovarian malignancy. ${ }^{12}$ Risk for osteoporosis increases with age. Hui et al conducted a study on 521 women and found that increasing age was predictive of increased fracture risk. ${ }^{13} A$ patient's risk for fracture increases with age even at the same BMD or T Score. ${ }^{14}$ In a study by Siris et al on 20,000 postmenopausal women, as compared to women aged 50-54 the odds of having osteoporosis was 5.9 times higher in women aged $65-69$ and 14.3 folds higher in women aged $75-79 .{ }^{15}$ In our study also, of the 40 patients who were osteoporotic majority were more than 55 years old. Osteoporosis typically produces no or subtle symptoms and at times the first manifestation of the disease is a low impact fracture. Back pain is a major symptom and signifies vertebral compression fractures. Approximately $50 \%$ of women over 65 years have spinal compression fractures leading to back pain. ${ }^{16}$ In our study backache was the commonest symptom and was present in $63.3 \%$ of the patients.

Bone density measured at the femoral neck by DXA is the best predictor of hip fracture. QUS is an inexpensive method of assessing bone quality, while QUS measurements do not correlate well with DXA measurements a $T$ score in the osteoporotic range on either test is associated with a high probability of hip fracture. ${ }^{17}$ Hans et al conducted a prospective study to assess the value of BMD measurements by ultrasound in predicting the risk for hip fracture and concluded that the os calcis ultrasound measurements were able to predict the risk of hip fracture in elderly women. ${ }^{18}$

\section{CONCLUSIONS}

QUS uses high frequency sound waves to measure bone density. It is cheap, radiation free and quick method of measuring bone density and is a good screening procedure. The results of the study show that $90 \%$ of the postmenopausal women had subnormal $\mathrm{T}$ scores. We recommend screening of postmenopausal women to identify osteoporosis in order to provide appropriate therapy to decrease the risk of fracture.

\section{REFERENCES}

1. Kanis JA, Melton LJ, Christiansen $C$ et al. The diagnosis of osteoporosis. J Bone Miner Res 1994; 9:1137-41.

2. NIH Consensus Development Panel on Osteoporosis prevention, diagnosis, and therapy. JAMA 2001; 285:785-95.

3. National osteoporosis foundation 2003 Disease statistics. Available at http://www.nof.org

4. Chrischilles EA, Butler CD, Davis CS et al. A model of lifetime osteoporosis impact. Arch Intern Med 1991; 151: 2026-32.

5. Johnell $O$. The socioeconomic burden of fractures. Today and in the $21^{\text {st }}$ century. Am J Med 1997; 103: 20-5s

6. Kanis JA. Assessment of fracture risk and its application to screening for postmenopausal osteoporosis: synopsis of a WHO report. Osteoporosis Int 1994; 4: 368-81.

7. Burger $\mathrm{H}$, DeLaet $\mathrm{CE}$, Weel $\mathrm{AE}$ et al. Added value of bone mineral density in hip fracture risk scores. Bone 1999; 25: 69-374.

8. Bouxsein ML, Radloff SE. Quantitative ultrasound of the calcaneus reflects the mechanical properties of calcaneal trabecular bone. J Bone Miner Res 1997; 12: 839-46.

9. Nelson $\mathrm{HD}$, Helfand $\mathrm{M}$, Woolf $\mathrm{SH}$ et al. Screening for postmenopausal osteoporosis: A review of the evidence for the US preventive services task force. Ann Intern Med 2002; 137: 529-41.

10. Consensus Development Conference V, 1993. Diagnosis, prophylaxis, and treatment of osteoporosis. Am J Med 1994; 90: 646-50.

11. Cooper C, Campian G, Melton IJ. Hip fractures in the elderly: a world wide projection. Osteoporosis Int 1992; 2: 285-9.

12. Kumar R,Chaudhry LJ. Osteoporosis: Investigations helping early diagnosis. Orthopedics Today 2010; 12(1): 13-16.

13. Hui SL, Slemenda CW, Johnston CC Jr. Age and bone mass as predictors of fracture in a prospective study. J Clin Invest 1988; 81 1804-9.

14. Kanis JA, Johnell O, Oden A. Ten year probabilities of osteoporotic fractures according to BMD and diagnostic thresholds. J Miner Res 2001; 16: S194

15. Siris ES, Miller PD, Barrett-Connor E et al. Identification and fracture outcomes of undiagnosed low bone mineral density in postmenopausal women: Results from the national osteoporosis risk assessment. JAMA 2001; 286: 2815-22.

16. Patel AM, Khadilkar SS. Postmenopausal osteoporosis. Menopause. Jaypee publishers; 42-66.

17. Screening of osteoporosis in postmenopausal women. Recommendations and Rationale. US Preventive services task force.

18. Hans D, Dargent Molina, Schott AM, et al. Ultrasonographic heel measurements predict hip fracture in elderly women: the EPIDOS prospective study. Lancet 1996; 348: 511-4. 